\title{
NUMERICAL FORMULATION IN FINITE ELEMENTS OF COMPOSITE BEAMS
}

\author{
A. A. Diacenco ${ }^{1}$, S. S. C. Jr. ${ }^{1}$,B.Jorge ${ }^{1}$, M.P.L.A. Popovic ${ }^{1}$ \\ ${ }^{1}$ Mechaninal of Institute of Engineering, Federal University of Itajubá \\ (aadiacenco@yahoo.com.br)
}

\begin{abstract}
This paper is devoted to procedures for the finite element modeling of composite beams. The numerical analysis is developed using the Timoshenko Theory of the Beams, which is implemented element containing two nodes and two degrees of freedom per node. The paper is organized as follows: introductory comments are presented regarding the use of composite materials in engineering applications. Numerical simulations present the results of the static and dynamic behavior of the composite beams. Composite structures, finite element modeling, timoshenko beams.
\end{abstract}

Keywords: Composite Structures, Finite Element Modeling, Timoshenko Beams.

\section{INTRODUCTION}

Composite materials have been increasingly used in various types of engineering systems, especially in aerospace structures, in which structural components must be designed to withstand harsh static and dynamic loading conditions, with typically high reliability levels ${ }^{[1,2]}$. In applications in which dynamic loads are involved, the interest in achieving as vibration amplitudes are directly related to fatigue and, as a result, to structural integrity. The great variety of materials properties and structural configurations makes the numerical modeling of the mechanical behavior of composite structures a complex task. This is a reason for which in the last decades, a great deal of effort has been devoted to the development of finite element models for characterizing the mechanical behavior of such materials, accounting for its typical variations of constructions and various orientations possibilities. Much of the knowledge available to date is compiled in the works by Reddy (1997).

\section{FINITE ELEMENT FORMULATION OF COMPOSITE BEAMS}

The mechanical behavior of the composite structure beam can be model by using the TimoshenkoTheory, in which the displacements at an arbitrary point in such a composite is expressed as follows:

$$
\begin{gathered}
u=-y \theta_{x} \\
w=w_{0}
\end{gathered}
$$


where $u$ e $w$ denote the displacements in directions $x$ and $y$. respectively. And $\theta_{x}$ is the crosssection rotation y direction.

The Timoshenko beam formulation accounts for transverse shear deformation. It is therefore capable of modeling thin and thick beams ${ }^{[3]}$. The figure shows the Timoshenko beam element with two nodes.

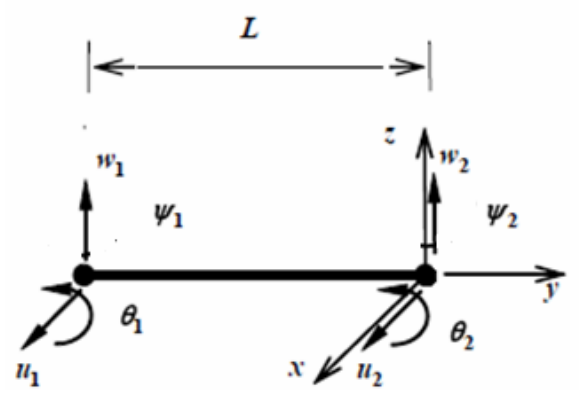

Figure 1. Beam element.

The Equation (1) can be rewritten:

$$
V(x, y, z, t)=\boldsymbol{A}(y) v(x, y, t)
$$

In Equation (2):

$$
\begin{aligned}
& V(x, y, z, t)=\left[\begin{array}{ll}
u(x, y, z, t) & w(x, y, z, t)
\end{array}\right]^{T} \\
& A(y)=\left[\begin{array}{cc}
0 & -y \\
1 & 0
\end{array}\right] \\
& v(x, y, t)=\left[w_{0}(x, y, t) \theta_{x}(x, y, t)\right]^{T}
\end{aligned}
$$

The usual strain-displacement relations are used and the resulting strains are separated in bending and transverse shear strains, $\boldsymbol{\varepsilon}_{b}$ and $\boldsymbol{\varepsilon}_{s}$, respectively, as follows:

$\boldsymbol{\varepsilon}_{b}(x, y, z, t)=\left[\boldsymbol{D}_{1}\right] \boldsymbol{v}=\boldsymbol{D}_{\boldsymbol{b}} \boldsymbol{v}$

$\boldsymbol{\varepsilon}_{s}(x, y, z, t)=\left[\boldsymbol{D}_{2}\right] \boldsymbol{v}=\boldsymbol{D}_{s}(y) \boldsymbol{v}$

Where: $v=\left[\begin{array}{ll}w_{0} & \theta_{x}\end{array}\right]^{T}$. And the matrices $\boldsymbol{D}_{i}$ are formed by differential operators appearing in the strain-displacement relations. 
The relation between strain and displacement was obtained the follows expressions:

$\varepsilon_{x}=y \frac{d \theta_{x}}{d x}$

$\gamma_{x z}=\frac{d w}{d x}+\theta_{x}$

Discretization of the displacement variables is made by using appropriate interpolation functions. Hence, for the two node element, the 2 mechanical variables included in vector $v$ are interpolated from their corresponding 4 nodal values through the following relation:

$$
v(\xi, t)=N(\xi) v(t)
$$

where:

$N(\xi)$ of dimensions $2 \times 2$, is the matrix formed by the beam element shape interpolation functions formulated in local coordinates $(\xi),-1 \leq \xi \leq 1$.

By associating Equations. (1), (2), (3), (4) and (7), the displacement and strain fields are found to be expressed in terms of the nodal values as follows:

$$
\begin{aligned}
& V(x, y, z, t)=\boldsymbol{A}(z) \boldsymbol{N}(\xi) v(t) \\
& \boldsymbol{\varepsilon}_{b}=\boldsymbol{D}_{b} \boldsymbol{N}(\xi) v=\boldsymbol{B}_{b}(\xi) v \\
& \boldsymbol{\varepsilon}_{s}=\boldsymbol{D}_{s} \boldsymbol{N}(\xi) v=\boldsymbol{B}_{s}(\xi) v
\end{aligned}
$$

Based on the stress-strain relations, the strain and kinetic energies of the composite beams can be formulated in terms of the natural variables of strain field and the mechanical material properties. After, Lagrange's equations are used, considering the nodal displacements and rotations as generalized coordinates, to obtain the following elementary mass and stiffnesses matrices, respectively:

$$
\begin{aligned}
& \boldsymbol{M}^{(e)}=\int_{-1}^{1}(\rho A) \boldsymbol{N}^{T}(\xi) \boldsymbol{N}(\xi)(\boldsymbol{J}) d \xi+\int_{-1}^{1}(\rho I) \boldsymbol{N}^{T}(\xi) \boldsymbol{N}(\xi)(\boldsymbol{J}) d \xi \\
& \boldsymbol{K}_{b}^{(e)}=\sum_{k=1}^{n} \int_{-1}^{1} \boldsymbol{B}_{b}^{T}(\xi) \boldsymbol{C}_{b}^{(k)}\left(\theta_{k}\right)(\boldsymbol{E I}) \boldsymbol{B}_{b}(\xi)(\boldsymbol{J}) d \xi \\
& \boldsymbol{K}_{s}^{(e)}=\sum_{k=1}^{n} \int_{-1}^{1} \boldsymbol{B}_{s}^{T}(\xi) \boldsymbol{C}_{s}^{(k)}\left(\theta_{k}\right)(\boldsymbol{G A}) \boldsymbol{B}_{s}(\xi)(\boldsymbol{J}) d \xi
\end{aligned}
$$

where I, G, E, A are, respectively, inertia moment, shear modulus, elastic modulus and area. 
In Equations. (10-12) $\operatorname{det}(\boldsymbol{J})$ indicates the determinant of the Jacobian of the transformation from the in-plane physical variables $(x, y)$ to the natural variables $(\xi)$, and matrices $\boldsymbol{C}_{b}(\theta)$ and $\boldsymbol{C}_{s}(\theta)$ represent, respectively, the orthotropic bending and shear elastic matrices, which are constructed according to the Classical Laminate Theory (CLT) as follows:

$$
\begin{aligned}
& \boldsymbol{C}_{b}(\theta)=\boldsymbol{T}_{b}(\theta) \overline{\boldsymbol{C}}_{b} \boldsymbol{T}_{b}^{T}(\theta) \\
& \boldsymbol{C}_{s}(\theta)=\boldsymbol{T}_{s}(\theta) \overline{\boldsymbol{C}}_{s} \boldsymbol{T}_{s}^{T}(\theta)
\end{aligned}
$$

where $\overline{\boldsymbol{C}}_{b}$ and $\overline{\boldsymbol{C}}_{s}$ are, respectively, the bending and shear elastic property matrices, referred to its principal orthotropic axis, and $\boldsymbol{T}_{b}(\theta)$ and $\boldsymbol{T}_{s}(\theta)$ are the associated rotation matrices [2,5].

From the elementary matrices computed for each element of the finite element mesh, the global equations of motion are constructed, accounting for the node connectivity, using standard finite element assembling procedures ${ }^{[4]}$. After assembling, the global equations of motion in the time domain can be written as follows:

$\boldsymbol{M} \ddot{\boldsymbol{q}}(t)+\boldsymbol{K q}(t)=\boldsymbol{f}(t)$

where $\boldsymbol{M}=\bigcup_{e=1}^{\text {nelem }} \boldsymbol{M}^{(e)}$ and $\boldsymbol{K}=\bigcup_{e=1}^{\text {nelem }} \boldsymbol{K}^{(e)}$ are the global FE mass and stiffness matrices. Symbol $\bigcup$ indicates matrix assembling and $\boldsymbol{q}(t)$ is the vector of global d.o.f's. $\boldsymbol{f}(t)$ is the vector of generalized external loads.

\section{NUMERICAL RESULTS}

\subsection{Analysis of frequence response functions (FRF's)}

For this application the first number is considered a beam in discretized in 20 element and it was considered that the fibers of the composite beam are oriented at an angle of the $30^{\circ}$.

Table1. Geometric characteristics and properties of the beam

\begin{tabular}{cc}
\hline Data & Value \\
\hline Length & $2 \mathrm{~m}$ \\
thickness & $0.1 \mathrm{~m}$ \\
inertia moment & $\left(\mathrm{h}^{3}\right) / 12$ \\
density & $1566 \mathrm{~kg} / \mathrm{m}^{3}$ \\
Poisson ratio & 0.30 \\
elastic modulus & $6,89 \mathrm{GPa}$ \\
shear modulus & $3,45 \mathrm{GPa}$ \\
\hline
\end{tabular}


The Figures 2 and 3 shows the FRF amplitudes of the composite material obtained through the implementation. Note that the theory used satisfactorily describes the FRF amplitudes. The thickness of the beam is a design parameter influence on the analysis of beams which can be seen through the FRF of Figure 3, which has changed the value of the thickness to 0.01 .

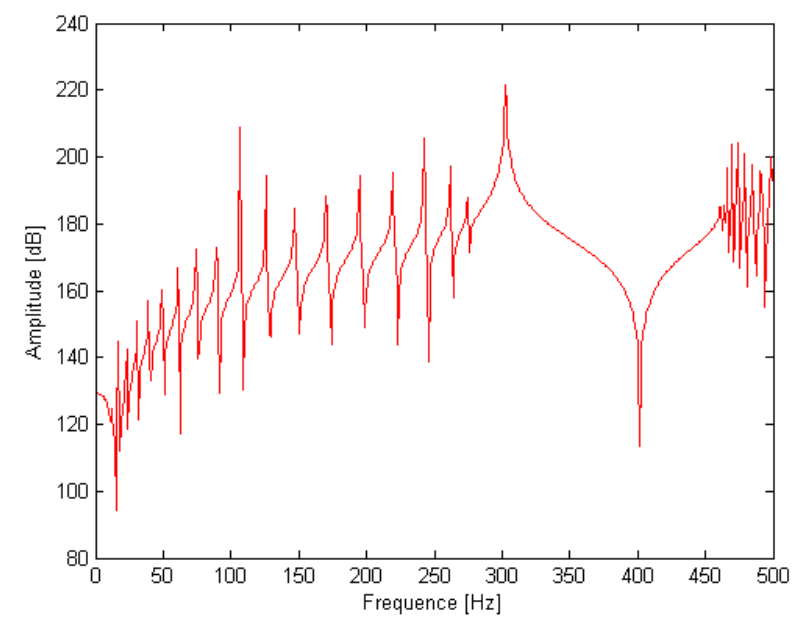

Figure 2. FRF amplitudes for $\mathrm{h}=0.1 \mathrm{~m}$.

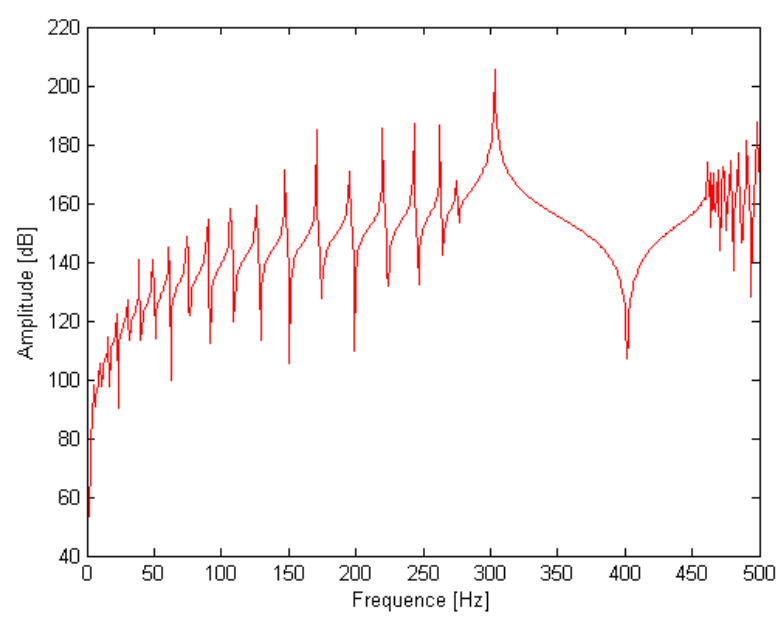

Figure 3. FRF amplitudes for $\mathrm{h}=0.01 \mathrm{~m}$.

\section{CONCLUSIONS}

The modeling procedure examined and developed in this work allowed to obtain the frequency response functions satisfactorily. It can be concluded also that the thickness of the beam so significant influence on the structural behavior. For future work is suggested incorporating a viscoelastic core to attenuate the vibration.

\section{Acknowledgements}

The authors are deeply grateful to the following organizations:

_ Minas Gerais State Agency FAPEMIG for the financial support to their research activities. 
CAPES Foundation, from the Brazilian Ministry of Education, for the financial support to their research activities.

\section{REFERENCES}

[1] Reddy JN. Mechanics of laminated composite plates: theory and analysis. 2nd ed. CRC Press; 1997.

[2] Diacenco A. A. Modelagem por Elementos Finitos de Materiais Compósitos Incorporando Material Viscoelástico para o Controle Passivo de Vibrações e Ruído, M.Sc.Dissertation, UNIFEI, Itajubá, 2010.

[3] LEE, J.; SCHULZ, W.W. Eigenvalue Analysis of timoshenko beams and axisymmetric mindlin plates by the pseudospectral method. Journal of Sound and Vibration, 269(3-4):609621,2004 .

[4] Huebner KH, Thornton EA. The finite element method for engineers. New York: John Wiley \& Sons; 1982.

[5] Diacenco, A. A.; de Lima, A. M. G.; Côrrea, E. O. Numerical Formulation In Finite Elements Of Damping In Composites Materials Using Theories Of First And Higher Order. Iberian Latin American Congress on Computational Methods in Engineering (CILAMCE),2011, Ouro Preto -MG. 